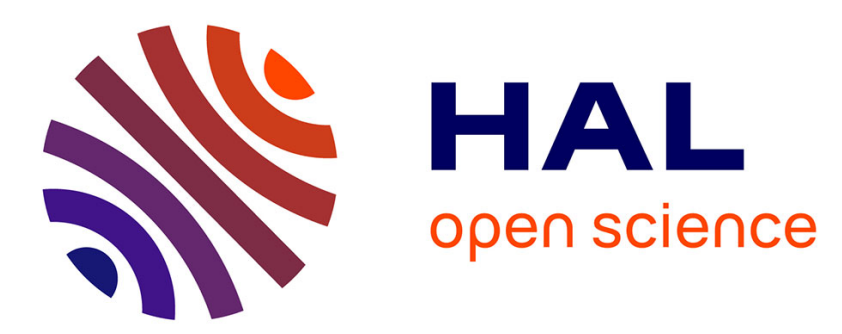

\title{
Piégeage d'électrons de très faible énergie pour la réalisation d'une source d'électrons polarisés de forte intensité
}

\author{
J.-M. Brebec, Ph. Coiffet, J.-P. Gilles
}

\section{To cite this version:}

J.-M. Brebec, Ph. Coiffet, J.-P. Gilles. Piégeage d'électrons de très faible énergie pour la réalisation d'une source d'électrons polarisés de forte intensité. Revue de Physique Appliquée, 1972, 7 (4), pp.339351. 10.1051/rphysap:0197200704033900 . jpa-00243637

\section{HAL Id: jpa-00243637 https://hal.science/jpa-00243637}

Submitted on 1 Jan 1972

HAL is a multi-disciplinary open access archive for the deposit and dissemination of scientific research documents, whether they are published or not. The documents may come from teaching and research institutions in France or abroad, or from public or private research centers.
L'archive ouverte pluridisciplinaire $\mathbf{H A L}$, est destinée au dépôt et à la diffusion de documents scientifiques de niveau recherche, publiés ou non, émanant des établissements d'enseignement et de recherche français ou étrangers, des laboratoires publics ou privés. 


\title{
PIÉGEAGE D'ÉLECTRONS DE TRÈS FAIBLE ÉNERGIE POUR LA RÉALISATION D'UNE SOURCE D'ÉLECTRONS POLARISÉS DE FORTE INTENSITÉ
}

\author{
J.-M. BREBEC, Ph. COIFFET et J.-P. GILLES \\ Institut d'Electronique Fondamentale, Laboratoire associé au CNRS \\ Université Paris-XI, Bâtiment 220, 91, Orsay, France
}

(Reçu le 17 mai 1972)

\begin{abstract}
Résumé. - Nous examinons d'abord les conditions nécessaires pour piéger une quantité importante d'électrons lents dans un système électromagnétique et pour leur transférer la polarisation d'un faisceau connu d'atomes orientés.

Nous proposons deux configurations permettant d'obtenir un faisceau polarisé d'électrons libres de forte intensité.

Une première, d'inspiration classique dont nous avons modifié le système d'injection afin d'augmenter ses performances.

Une seconde, utilisant un système magnéto-électrique original, pouvant donner un courant crête supérieur à $1 \mathrm{~mA}$ (plus de $10^{9}$ électrons par pulse), avec une polarisation voisine de l'unité $(0,9)$.

Abstract. - We first examine the necessary conditions for collecting many slow electrons in a magnetic and electric trap and to transfer to them the polarisation of a well known oriented atomic beam.

We propose two field configurations which permit one to obtain a polarised free electron beam of strong intensity.

The first configuration results in a modification of an injection system to improve its performance.

The second, uses an original magneto electric system which would be able to give more than

$1 \mathrm{~mA}$ peak current (more than $10^{9}$ electrons per pulse) with a polarization near unity $(0.9)$.
\end{abstract}

1. Le piégeage. Principe de base. - Un champ magnétique longitudinal et deux miroirs électrostatiques placés perpendiculairement à l'axe et aux lignes de champ magnétique constituent l'élément de base de nombreux pièges à électrons.

Dans la pratique, l'injection des électrons dans le piège pose un problème délicat à résoudre puisque si ceux-ci ne peuvent entrer dans la trappe, ils ne peuvent également en sortir. Lorsque les électrons sont assez énergétiques (énergie cinétique $E$ ) et le champ magnétique $B$ assez faible pour que le rayon de giration des électrons soit de l'ordre de grandeur du rayon géométrique du piège, on injecte les particules suivant une direction radiale dans une zone de champ magnétique nul. Les particules s'appuient dans leur mouvement sur des surfaces limites, de cette façon, la probabilité de fuite par la zone d'injection est très faible [1].

Cette méthode n'est plus utilisable si les particules ont une énergie cinétique de quelques électrons-volts et si le champ magnétique est fort (c'est-à-dire lorsque la quantité $\left(2 \mathrm{mE} / e B^{2}\right)^{1 / 2}$ représentant le rayon de giration maximum de l'électron est de l'ordre de grandeur du millimètre). Les particules suivent alors approximativement les lignes de champ magnétique dans une zone de potentiel électrique constant.
Trois solutions peuvent alors être retenues :

a) La création des électrons à piéger à l'intérieur même du piège, en ionisant des atomes neutres (vapeur ou jet atomique). Le rendement dépend de la section efficace d'ionisation et de la densité des particules incidentes et cibles. Lorsque cette densité de molécules ou d'atomes doit être très petite pour respecter certains impératifs expérimentaux, le rendement est mauvais et le nombre d'électrons piégés reste extrêmement faible.

b) L'ouverture de la barrière de potentiel à l'une des extrémités de la trappe durant l'injection, effectuée suivant l'axe. On referme cette barrière dès que les particules s'y représentent, après avoir été réfléchies par l'autre miroir [2], [3]. Ceci permet seulement d'emprisonner les électrons émis pendant le temps d'un aller-retour, ce qui limite énormément les performances, surtout si l'on désire conserver des électrons d'énergie cinétique inférieure à $10 \mathrm{eV}$, comme nous le verrons plus loin. La faible émissivité des cathodes des canons fonctionnant dans cette gamme d'énergie constitue un handicap supplémentaire. Le courant moyen extrait d'un tel piège ne dépasse pas la dizaine de nanoampères [4]. 
c) Krisciokaitis [5] a proposé une méthode originale permettant d'injecter des électrons pendant $400 \mu \mathrm{s}$, les potentiels des électrodes variant de telle façon que :

- Les électrons soient toujours soumis à une force dirigée vers l'intérieur du piège. (Ils sont alors piégés sur une longueur de $1,10 \mathrm{~m}$.)

- L'énergie cinétique des électrons soit inférieure à $10 \mathrm{eV}$.

Les performances du système sont là aussi limitées par la faible émissivité de la cathode. Mais en plus, l'intérieur du piège est recouvert d'une couche isolante de fluorocarbone afin d'éviter la dépolarisation du faisceau d'atomes d'hydrogène, qui est simultanément envoyé dans ce piège. Cet isolant risque de se charger et de perturber singulièrement l'injection.

L'auteur pense piéger $10^{11}$ électrons dans son volume. Pour ce faire, il lui est nécessaire de vaincre les forces dues à la charge d'espace. L'examen de la méthode utilisée pour vaincre ces forces ne nous permet pas de dégager les mêmes conclusions : le calcul semble trop optimiste. La limite imposée par l'effet de la charge d'espace est de $10^{9}$ particules dans le piège. Nous ne considérerons que cette valeur à titre de comparaison avec les résultats obtenus dans l'étude que nous présentons ici.

Dans ce qui suit, nous proposons deux procédés, dont l'un permet d'atteindre les mêmes ordres de grandeur que la méthode de Krisciokaitis. Ceci nous permettra de faire l'échange de spin entre les électrons et des atomes de potassium polarisés [6], [7] et de fournir ainsi une source d'électrons polarisés délivrant un faisceau d'intensité suffisante pour pouvoir envisager son injection dans un accélérateur linéaire d'électrons, ou diverses expériences de physique atomique.

Nous allons tout d'abord étudier les différentes conditions à respecter pour que l'échange de spin ait lieu effectivement entre des électrons et des atomes polarisés.

Nous exposerons ensuite les deux méthodes étudiées qui permettent de respecter ces conditions.

2. Conditions à respecter pour réaliser des collisions électron-atome avec échange de spin. - 2.1 CONDITION SUR L'ÉNERGIE DES ÉLECTRONS. - La section efficace d'échange de spin décroît rapidement lorsque l'énergie cinétique $E$ des électrons augmente [8]: d'abord comme $1 / E$ puis plus rapidement lorsque $E$ approche de l'intervalle d'énergie singulet-triplet des états liés de l'ion alcalin négatif qui se crée. Pour le potassium, cet intervalle est de $0,3 \mathrm{eV}$. En fait, il a été montré expérimentalement [9], [10] que jusqu'à $9 \mathrm{eV}$, la section efficace variait peu et restait voisine de $2 \times 10^{-14} \mathrm{~cm}^{2}$ pour le potassium.

Nous nous fixerons donc une gamme d'énergie telle que $E \leqslant 9 \mathrm{eV}$.
2.2 CONDITIONS SUR LE TEMPS DE « CONTACT » ENTRE LES ÉLECTRONS ET LES ATOMES. - Le temps de contact n'est autre que celui nécessaire à un nombre de collisions assez grand d'un même électron avec divers atomes polarisés, de telle sorte que la probabilité de transfert de la polarisation du faisceau d'atomes au nuage d'électrons soit voisine de l'unité.

L'évolution de la polarisation $P_{\mathrm{e}}$ du nuage d'électron en fonction de la polarisation $P_{\mathrm{A}}$ du faisceau atomique. $P_{\mathrm{A}}$ est donné par la relation [11] :

$$
P_{\mathrm{e}}=P_{\mathrm{A}}\left(1-\mathrm{e}^{-t / T}\right)\left(\frac{1+T}{\tau}\right) .
$$

Dans cette formule, nous avons $\tau$ (temps de relaxation de dépolarisation par choc entre les électrons nouvellement polarisés et les molécules ou atomes résiduels) qui est très supérieur à $T$ (temps de relaxation d'échange de spin), si le vide est suffisamment bon.

En appliquant les lois statistiques, nous avons:

$$
T=\frac{1}{2 \sigma_{\mathrm{es}} \rho \bar{v}_{\mathrm{e}}}
$$

où :

$\sigma_{\mathrm{es}}$ est la section efficace d'échange de spin entre électrons et atomes. Sa valeur est voisine de $2 \times 10^{-14} \mathrm{~cm}^{2}$ pour des collisions avec échange de spin entre des électrons d'énergie cinétique inférieure à $9 \mathrm{eV}$ et des atomes de potassium orientés.

$\rho$ est la densité des atomes de potassium dans le faisceau. Sa valeur est supérieure à $2 \times 10^{9}$ atomes $/ \mathrm{cm}^{3}$ en ce qui concerne le faisceau d'atomes de potassium déjà réalisé [7] et peut être accrue jusqu'à $10^{10}$ ato$\mathrm{mes} / \mathrm{cm}^{3}\left(^{*}\right)$.

$\bar{v}_{\mathrm{e}}$ est la vitesse moyenne d'un électron (approxinativement $1,7 \times 10^{6} \mathrm{~m} / \mathrm{s}$ pour des électrons uniformément répartis entre 1 et $10 \mathrm{eV}$ ).

Avec ces données, nous trouvons : $T=12 \mu \mathrm{s}$.

Dès que $t \geqslant 3 T$, nous pouvons considérer que $P_{\mathrm{e}} \simeq P_{\mathrm{A}}$, soit donc au bout de $36 \mu$ s. Mais dans le piège, les électrons dans leur mouvement de va et vient, ne sont en contact avec les atomes de potassium que pendant $1 / 20^{\mathrm{e}}$ du temps (les deux faisceaux seront croisés). Avec un temps de piégeage de $1000 \mu \mathrm{s}$, le transfert quasi total de polarisation doit être assuré.

(*) En référence [7], le but était d'obtenir des électrons polarisés en ionisant un faisceau d'atomes de potassium polarisés par un rayonnement UV. Le pourcentage des molécules dans le faisceau étant de $3 \%$ entre 350 et $400^{\circ} \mathrm{C}$ et la section efficace de photoionisation des molécules étant 4000 fois plus grande que celle des atomes, nous étions obligés de nous limiter à une température de source égale à $300-310^{\circ} \mathrm{C}$. Dans le cas présent la température du four pourra être portée à $T=400^{\circ} \mathrm{C}$.

1) La vitesse des atomes variant peu, $\sigma_{\mathrm{es}}$ gardera la même valeur.

2) La densité des molécules étant très faible $\left(d<10^{7} \mathrm{~mol} / \mathrm{cm}^{3}\right.$, soit $P<10^{-12} \mathrm{~mm} \mathrm{Hg}$ ), les chocs dépolarisants sur ces molécules seront pratiquement inexistants. Dans ces conditions, nous pouvons obtenir aisément une densité de $10^{10}$ atomes $/ \mathrm{cm}^{3}$. 
2.3 CONDITIONS SUR LE CHAMP MAGNÉTIQUE. - Les électrons faisant un grand nombre d'aller-retour dans le piège, la composante radiale du champ magnétique risque d'avoir une influence importante sur la dépolarisation des électrons. On donne, en annexe, le calcul de cette influence évaluée de la façon suivante :

L'électron, au cours de son mouvement, est placé dans un champ magnétique $H_{z}$ constant et dans un champ $H_{r}$, perpendiculaire à $H_{z}$, et oscillant à la fréquence $\omega / 2 \pi$ correspondant aux aller-retour des électrons dans le piège (perturbation dépendant du temps).

Si l'on veut que la dépolarisation $\Delta P$ due au champ magnétique seul, sans tenir compte des facteurs de relaxation, soit telle que :

$$
\frac{\Delta P}{P}<10^{-3}
$$

la condition sur le champ radial est alors :

$$
\frac{H_{r}}{H_{z}}<2,2 \times 10^{-2} .
$$

Pour un champ magnétique de 125 Oe, le champ radial maximum dans la zone utile du piège doit être inférieur à 2,8 Oe.

2.4 CONDITIONS A IMPOSER AU VIDE. - Les chocs des électrons sur les molécules de gaz résiduel peuvent avoir deux conséquences :

a) Ils peuvent induire un renversement de spin pour les électrons déjà polarisés. Il faut donc que le libre parcours moyen $L$ d'un électron dans le gaz résiduel soit supérieur à la distance $d$ que cet électron parcourt pendant le temps de piégeage conduisant à l'échange de spin.

Nous savons que :

$$
L=\frac{1}{\pi a^{2} \rho_{r}}
$$

$a$, étant le rayon classique de l'électron $\left(2 \times 10^{-8} \mathrm{~cm}\right)$ et $\rho_{r}$ le nombre de particules de gaz résiduel par $\mathrm{cm}^{3}$, pour avoir $L$ en $\mathrm{cm}$ :

$$
L=\frac{8 \times 10^{14}}{\rho_{r}} .
$$

Les électrons ayant une énergie cinétique inférieure à $9 \mathrm{eV}$, leur vitesse est inférieure à $2 \times 10^{8} \mathrm{~cm} / \mathrm{s}$, donc la distance maximum parcourue pendant la durée du piégeage $(1000 \mu \mathrm{s})$ est de $10^{5} \mathrm{~cm}$. Nous devons donc avoir :

$$
\rho_{r}<8 \times 10^{9} \text { particules } / \mathrm{cm}^{3},
$$

ce qui nous conduit à la température ordinaire de $300{ }^{\circ} \mathrm{K}$ à une pression de gaz résiduel $P_{r}$, telle que :

$$
P_{\boldsymbol{r}}<25 \times 10^{-8} \mathrm{~mm} \mathrm{Hg} .
$$

Nous nous sommes donc imposés :

$$
P_{r} \simeq 10^{-8} \mathrm{~mm} \mathrm{Hg}
$$

soit

$$
\rho_{r} \simeq 0,3 \times 10^{9} \text { particules } / \mathrm{cm}^{3} .
$$

Remarquons que la section efficace d'échange de spin $\left(\sigma_{\mathrm{es}}=2 \times 10^{-14} \mathrm{~cm}^{2}\right)$ est très supérieure à celle des chocs de plein fouet entre électrons et atomes $\left(\sigma=10^{-16} \mathrm{~cm}^{2}\right)$, ce qui permet en théorie d'avoir une pression de gaz résiduel presque aussi forte que la pression prévue dans le jet d'atomes polarisés.

b) Les chocs peuvent réduire la durée de vie de l'électron dans le piège s'ils s'effectuent de telle sorte que les électrons soient diffusés sur les parois. Essayons de déterminer un ordre de grandeur de cette durée de vie. Faisons pour cela les hypothèses suivantes:

1. L'énergie cinétique transversale de l'électron due à son mouvement de rotation, est beaucoup plus grande que son énergie longitudinale. Le rayon de giration maximum dans un champ magnétique de $10^{-2} \mathrm{~T}$ est alors de $R=1 \mathrm{~mm}$.

2. L'électron subit des chocs de plein fouet qui inversent le sens de sa vitesse et provoquent un déplacement radial de la quantité $2 R$. La vitesse moyenne de diffusion est donc égale à :

$$
\bar{v}_{\mathrm{d}}=\frac{2 R}{T_{r}}
$$

où $T_{r}$, temps séparant deux chocs successifs, est donné par :

$$
T=\left(\frac{2 \pi}{\omega_{\mathrm{L}}}\right) \times\left(\frac{L}{2 \pi R}\right)=\frac{L}{R \omega_{\mathrm{L}}}
$$

où $\omega_{L}$ est la fréquence de Larmor $\left(\omega_{L}=e B / 2 m\right)$.

Le coefficient de diffusion $D$ s'écrit donc :

$$
D=R \bar{v}_{\mathrm{d}}=2 \pi \rho_{r} a^{2} \omega_{\mathrm{L}} R^{3}
$$

l'équation de la diffusion étant :

$$
D \cdot \operatorname{div}(\operatorname{grad} \Phi)=\frac{\partial \Phi}{\partial t}
$$

où $\Phi$ est la densité de particules, nous avons :

$$
\Phi(r, t)=\left(\frac{1}{t}\right) \exp \left[-\frac{r^{2}}{4 D t}\right] .
$$

La durée de vie des électrons pour une paroi qui absorbe ceux-ci au rayon $r=b$, est alors :

$$
t_{\mathrm{v}}=\frac{b^{2}}{4 D}=\frac{b^{2}}{8 \pi R^{3} \rho_{r} \omega_{\mathrm{L}} a^{2}}
$$

avec les valeurs obtenues précédemment et en supposant $b / R=10$, nous avons :

$$
t_{\mathrm{v}}=150 \mathrm{~ms} \text {. }
$$

C'est la durée de vie minimum dans le cas le plus défavorable, en $1 \mathrm{~ms}$, on peut donc supposer les pertes comme négligeables.

Les quatre conditions que nous venons d'exposer sont impératives pour l'application qui nous intéresse : 
la production d'électrons polarisés par échange de spin. Elles dirigeront donc notre projet de piège à électrons dans ce qui suit.

3. Calcul de la configuration magnétique. - Les dimensions de la zone de piégeage, de forme cylindrique, à symétrie axiale, ont été choisies arbitrairement : longueur $20 \mathrm{~cm}$, diamètre $4 \mathrm{~cm}$. On veut que dans cette zone :

$$
\begin{gathered}
H_{2}=125 \mathrm{Oe} \\
H_{R}<1 \mathrm{Oe}, \text { limite que nous nous imposons } \\
\text { pour éviter tout phénomène de } \\
\text { dépolarisation. }
\end{gathered}
$$

Le champ magnétique est créé par deux bobines placées en position de Helmholtz. La distance qui les sépare doit être suffisante pour placer divers instruments. Le rayon intérieur doit être assez grand pour y placer une enceinte à vide contenant les électrodes de piégeage.

Une première approche de la solution a consisté à développer le champ en coordonnées sphériques, en utilisant les polynômes de Legendre [12], [13] (Fig. 1)

$$
\begin{aligned}
H_{z}(\rho, \theta)=0,4 \times & \pi \times j \times R_{2} \times \\
& \times\left\{\sum_{n=0} M_{2 n}\left(\frac{\rho}{R_{2}}\right)^{2 n} \times P_{2 n}(\cos \theta)\right\}
\end{aligned}
$$$$
H_{R}(\rho, \theta)=-0,4 \times \pi \times j \times R_{2} \times \sin \theta
$$$$
\times\left\{\sum_{n=1} N_{2 n}\left(\frac{\rho}{R_{2}}\right)^{2 n} \times P_{2 n}^{\prime}(\cos \theta)\right\}
$$

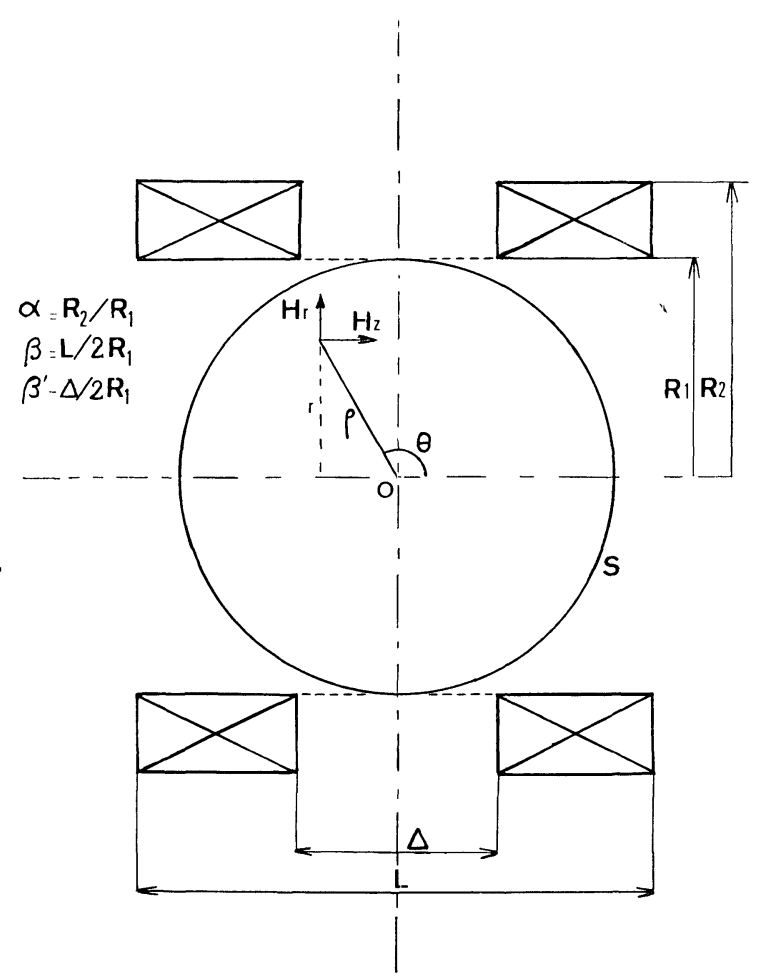

FIG. 1. - Définition des paramètres des bobines. où les $P_{i}$ et $P_{i}^{\prime}$ sont respectivement les polynômes de Legendre d'ordre $i$ et leur dérivée, $R_{2}$ est le rayon extérieur des bobines en $\mathrm{cm}, M_{i}$ et $N_{i}$ sont des coefficients sans dimension calculables en fonction des paramètres des bobines, $j$ est la densité de courant en $\mathrm{A} / \mathrm{cm}^{2}$ traversant chaque bobine et $H$ est en Oe.

Nous avons alors cherché pour quelle valeur de :

$\alpha$ : rapport du diamètre extérieur au diamètre intérieur,

$\beta$ : rapport de la longueur totale au diamètre intérieur,

$\beta^{\prime}$ : rapport de l'écartement des deux bobines au diamètre intérieur,

le champ est le plus homogène possible, en respectant la condition

$$
H_{R}<1 \text { Oe pour } H_{z} \text { au centre égal à } 125 \mathrm{Oe} \text {. }
$$

Le calcul n'est valable qu'à l'intérieur d'une sphère $S$ (Fig. 1) de rayon $R=R_{1}$ (rayon intérieur). On s'aperçoit que le développement converge àssez lentement; mais en considérant les quatre premiers termes de ce développement, nous avons pu déterminer facilement (calcul très rapide sur IBM J 75) les valeurs approchées des paramètres $\alpha, \beta$ et $\beta^{\prime}$.

Connaissant ces valeurs approchées, et voulant obtenir les valeurs du champ magnétique à une distance supérieure à $\rho=R_{1}$, nous avons utilisé un autre développement du champ magnétique.

Les formules en sont les suivantes :

$$
\begin{aligned}
& H_{z}(r, z)=H_{0}(z)+\sum_{p=1}^{\infty}\left(\frac{r}{2}\right)^{2 p} \frac{(-1)^{p}}{(p !)^{2}} \frac{\partial^{(2 p)} H_{0}(z)}{(\partial z)^{2 p}} \\
& H_{R}(r, z)=\sum_{p=0}\left(\frac{r}{2}\right)^{2 p+1} \frac{(-1)^{2 p+1}}{(p+1)(p !)^{2}} \frac{\partial^{(2 p+1)} H_{0}(z)}{(\partial z)^{2 p+1}}
\end{aligned}
$$

$H_{0}(z)$ représente le champ magnétique sur l'axe.

Ce calcul valable dans tout l'espace, contrairement au calcul précédent (sauf dans les zones où la densité de courant n'est pas nulle), a l'avantage d'être rapidement convergent dans le volume qui nous intéresse : les dérivées du champ ont été calculées jusqu'au $8^{\mathrm{e}}$ ordre. En réajustant très peu les paramètres $\alpha, \beta$ et $\beta^{\prime}$ les valeurs suivantes ont été retenues :

$$
\alpha=1,2, \quad \beta=1, \quad \beta^{\prime}=0,4 \text {. }
$$

Pour des raisons pratiques, nous avons choisi le rayon intérieur égal à $R_{1}=15 \mathrm{~cm}$, ce qui donne :

- rayon extérieur : $R_{2}=18 \mathrm{~cm}$;

- longueur totale : $L=30 \mathrm{~cm}$;

- écart entre les bobines : $L^{\prime}=12 \mathrm{~cm}$;

— densité de courant $: j=1 \mathrm{~A} / \mathrm{mm}^{2}$;

- champ magnétique au centre $: H_{0}(\mathrm{O})=125 \mathrm{Oe}$;

- champ radial dans la zone de piégeage : $H_{R}<1 \mathrm{Oe}$.

Le champ est connu avec une précision de l'ordre de $5 \%$ à une distance $r=8 \mathrm{~cm}$. Sur la figure 2 sont représentées les zones pour lesquelles $H_{R}<1$ Oe pour 


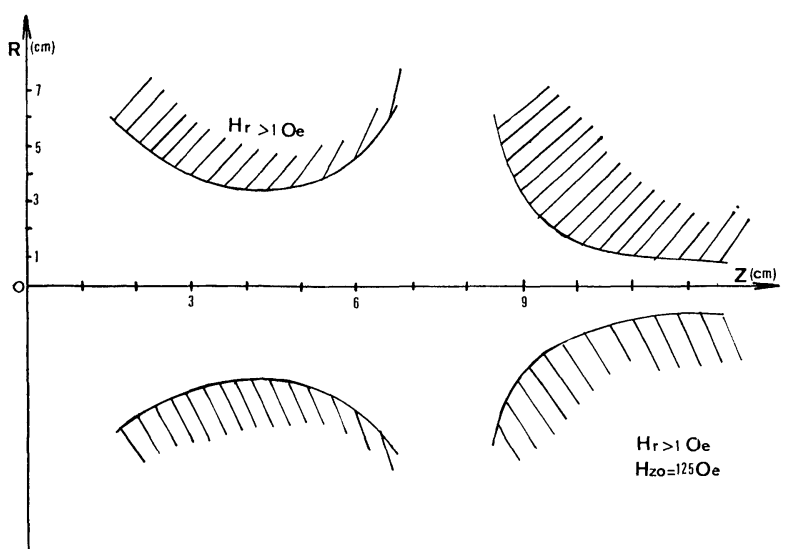

FIG. 2. - Région pour laquelle $H_{r}<1$ Oe pour $H_{z_{0}}=125 \mathrm{Oe}$; $\alpha=1,2 ; \beta=1,0 ; \beta^{\prime}=0,4$.

$H_{0}(\mathrm{O})=125$ Oe. On voit que l'on peut considérer le champ comme constant à mieux que $1 \%$ près dans toute la zone de piégeage. Les figures 3 et 4 donnent

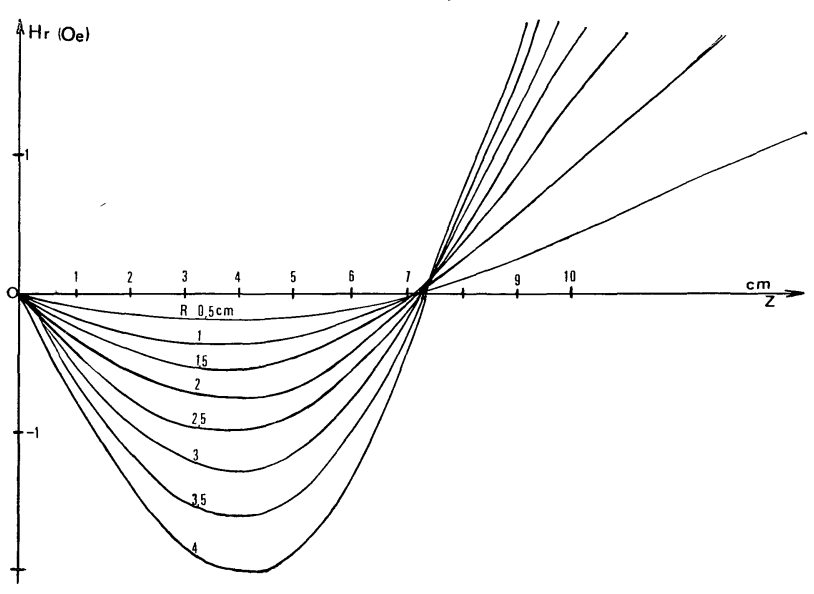

FIG. 3. $-H_{r}$ en fonction de $r$ et $z\left(\alpha=1,2 ; \beta=1,0 ; \beta^{\prime}=0,4\right)$.

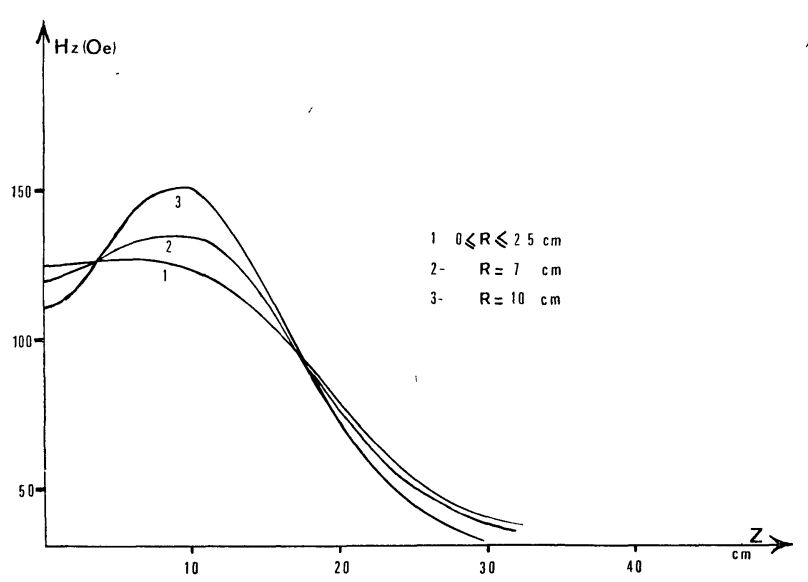

FIG. 4. $-H_{z}$ en fonction de $r$ et $z\left(\alpha=1,2 ; \beta=1,0 ; \beta^{\prime}=0,4\right)$;

$H_{R}$ et $H_{z}$ en fonction de $r$ et $z$. La figure 5 donne la forme des lignes de champ. Ceci est utile pour calculer la position du canon dont le rôle sera envisagé dans la suite $(\S 5.2 .1)$.

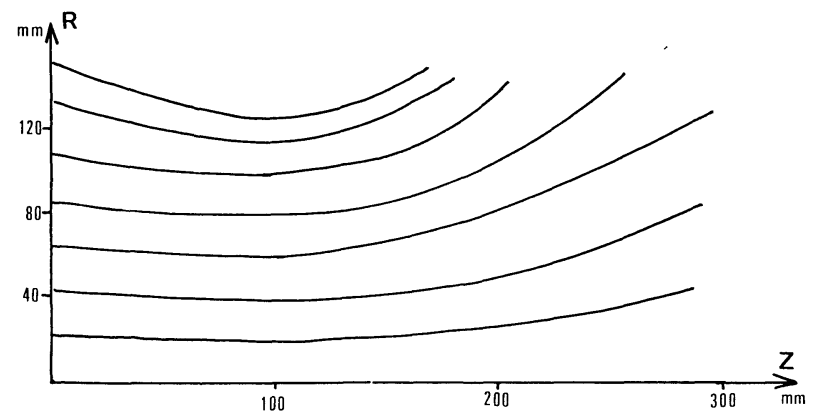

Fig. 5. - Forme des lignes de champ magnétique crées par les bobines $\left(\alpha=1,2 ; \beta=1,0 ; \beta^{\prime}=0,4\right)$.

4. Obtention des électrons de faible énergie. 4.1 A L'AIDE D'UN CANON. - a) La méthode la plus simple consiste à utiliser une cathode à oxydes et à en extraire les électrons par une anode portée au potentiel $\Phi_{0}$, par rapport à la cathode, correspondant à l'énergie désirée. La performance maximum s'obtient en formant un flux de Brillouin : cathode plane et anode cylindrique sont plongées dans une induction magnétique longitudinale dont la valeur est fonction $\mathrm{du}$ rayon $r_{0}$ du faisceau choisi.

$$
\begin{aligned}
\left(B_{z}\right)_{I_{\max }} & =\left(\frac{16 m \Phi_{0}}{3 e r_{0}^{2}}\right)^{1 / 2} \\
I_{\max } & =25,4 \times 10^{-6} \Phi_{0}^{3 / 2} .
\end{aligned}
$$

Considérant le cas où $\Phi_{0}=5 \mathrm{~V}$ et $B_{z}=125 \mathrm{G}$, nous devons avoir théoriquement :

$$
\begin{aligned}
& r_{0}=1 \mathrm{~mm} \\
& I=280 \mu \mathrm{A} .
\end{aligned}
$$

Dans la pratique, le faisceau n'est cylindrique que si $B_{z} \gg\left(B_{z}\right)_{I_{\max }}$ et $I \ll I_{\max }$, et il est prudent de diviser par un facteur 10 cette valeur de $I_{\max }$ afin d'obtenir un fonctionnement sûr selon cette méthode.

b) Une autre solution consisterait à ralentir le faisceau d'électrons rapides, dans une induction magnétique élevée : ceci afin de considérer que les électrons suivent les lignes de champ magnétique (hypothèse vérifiée pour des électrons de $10 \mathrm{eV}$ d'énergie cinétique dans une induction magnétique de $125 \mathrm{G}$ ).

Le schéma de principe en serait le suivant : les électrons émis par une cathode circulaire $(V=0)$, puis accélérés par une anode portée à une tension $V_{\mathrm{a}}$ sont ensuite ralentis par une électrode cylindrique portée à $V_{R}$ afin que sur l'axe les électrons aient une énergie cinétique $e V_{0}$ inférieure à $9 \mathrm{eV}$. On suppose que le canon a une pervéance de $10^{-6}$; le courant émis est donné par $I=10^{-6} V_{\mathrm{a}}^{3 / 2}$.

Supposons que $V_{R}$ soit le potentiel sur le rayon extérieur du faisceau, l'intensité maximum admise dans cette électrode supposée plongée dans un champ magnétique de valeur infinie est donnée par [14] :

$$
\begin{aligned}
& I_{\max }=32,4 \times 10^{-6} V_{R}^{3 / 2} \\
& V_{0}=0,174 V_{R} .
\end{aligned}
$$


Ainsi, si $V_{R}=5 \mathrm{~V}$, nous avons théoriquement :

$$
V_{0}=0,9 \mathrm{~V}
$$

et le courant $I_{\max }$ pourrait être fourni par un canon de pervéance $10^{-6}$ fonctionnant sous une tension $V_{\mathrm{a}}=50 \mathrm{~V}$. On a alors $: I=360 \mu \mathrm{A}$.

Mais cette limite ne pourrait être atteinte dans la pratique, l'induction magnétique imposée n'étant pas infinie.

4.2 Utilisation DE L'ÉMISSION SECONDAIRE. 4.2.1. - Une photocathode excitée par un rayonnement intense peut fournir un courant appréciable d'électrons de quelques eV d'énergie. Cependant les surfaces photo-émissives à grand rendement émissif sont particulièrement fragiles et difficiles à manipuler ; leurs caractéristiques évoluent avec le temps et il faut éviter les contacts avec l'air (fabrication et exploitation sous de très bons vides). Ces défauts nous ont conduit à éliminer cette solution.

4.2.2. - Une surface solide convenable soumise à un bombardement d'électrons réémet des électrons secondaires. Le rendement est fonction de la substance choisie et de l'énergie du faisceau primaire; il est presque toujours supérieur à l'unité pour une énergie primaire de l'ordre de 100 à $500 \mathrm{eV}$. Le spectre d'énergie de l'émission secondaire est quasi identique pour tous les corps et toutes les énergies primaires : les spectres d'énergie mettent en évidence une importante émission d'électrons secondaires vrais d'énergie 4-5 eV, et un second pic formé d'électrons primaires réfléchis (Fig. 6).

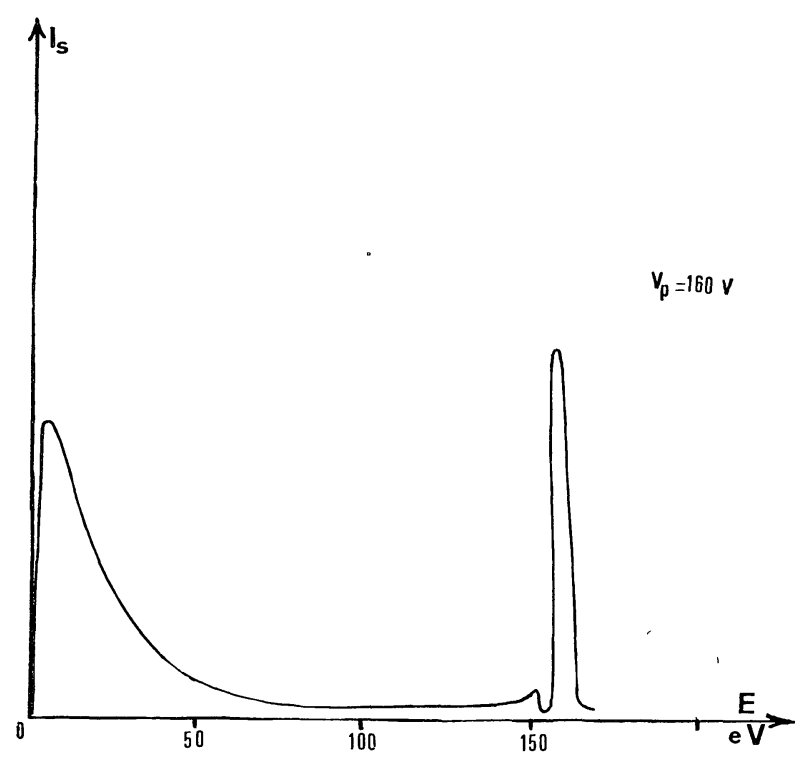

Fig. 6. - Spectre général d'énergie des électrons secondaires émis à l'aide d'un faisceau primaire accéléré sous $160 \mathrm{~V}$.

Ces électrons réfléchis seront éliminés du piège étudié car les barrières de potentiel établies aux extrémités sont insuffisantes pour les retenir.

Les substances qui possèdent des coefficients $\delta$ (rap- port de l'intensité secondaire à l'intensité primaire) très grands, sont, soit des alliages (comme $\mathrm{Ni}-\mathrm{Be}$ ), soit des substances composées ( $\mathrm{LiF}, \mathrm{NaCl}, \mathrm{SbCs}_{3}$, etc.). La plupart de ces corps sont des isolants, ce qui pose des problèmes de charge, ou sont fragiles (détérioration rapide, pas de remise à l'air, etc. comme pour les photocathodes).

Il semble donc que l'on ait intérêt à choisir un métal pur. Bruining [15] donne une liste des $\delta_{\max }$ et des énergies du faisceau d'électrons qui le fournit, pour un grand nombre de métaux et d'éléments (Fig. 7). Le

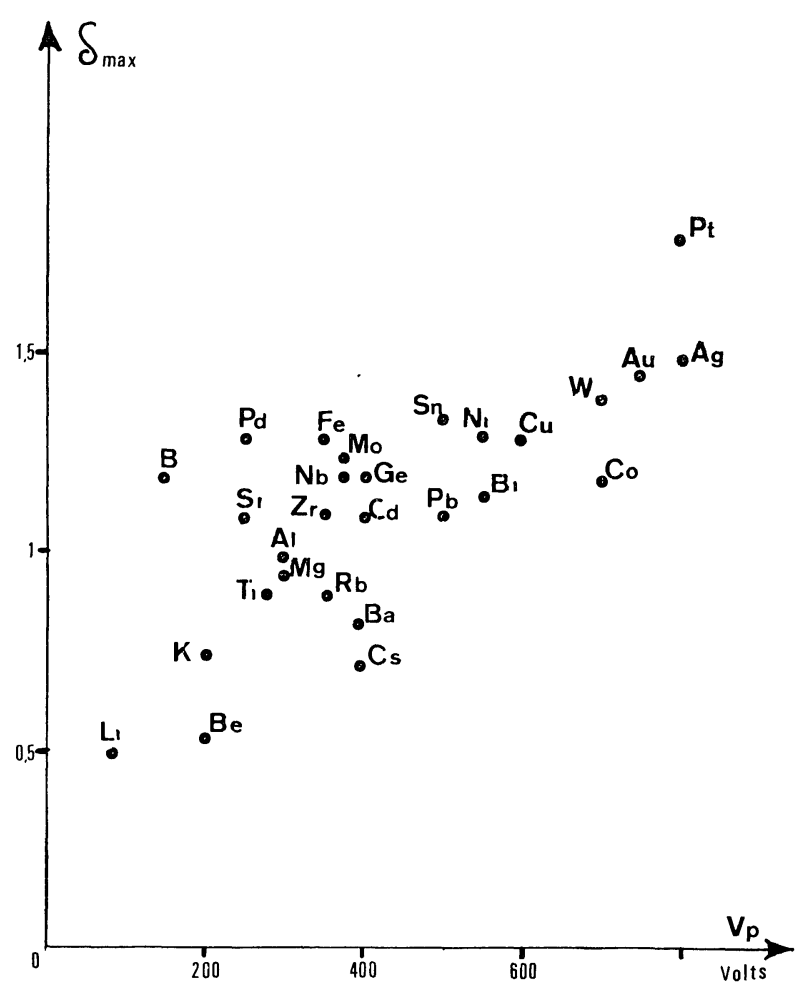

FIG. 7. - Coefficient d'émission secondaire $\delta$ maximum en fonction de l'énergie optimum des électrons primaires pour divers éléments.

meilleur rendement est donné par le platine bombardé par des électrons de $800 \mathrm{eV}(\delta=1,8)$. Sous cette tension on atteindra facilement la limite imposée pour la charge d'espace.

5. Les pièges à électrons proposés. - Nous décrivons ici deux types de solution possible.

5.1 Amélioration D'Une solution CLASSiQue [2], [3]. - 5.1.1 Description. Fonctionnement. - Le piège est constitué de trois cylindres plongés dans un champ magnétique homogène parallèle à leur axe (symétrie cylindrique) et portés à des potentiels alternativement positifs et négatifs (Fig. 8). Les électrons primaires, de $800 \mathrm{eV}$ d'énergie cinétique, traversent le piège et arrivent sur la pastille émissive $P$ (en platine) portée à un potentiel variant de +3 à $-10 \mathrm{~V}$. Cette pastille réémet des électrons secondaires de quelques électrons-volts d'énergie cinétique qui sont alors accé- 


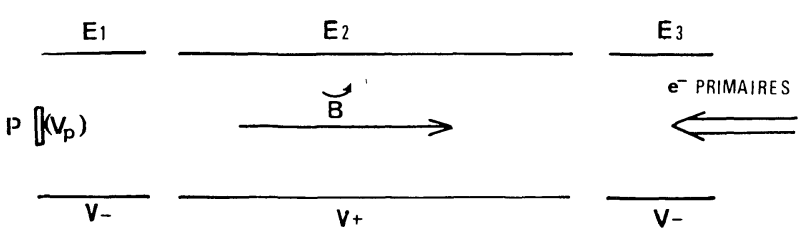

FIG. 8. - Trappe à trois électrodes.

lérés par l'électrode $\mathrm{E}_{1}\left(V_{\mathrm{E}_{1}}=0 \mathrm{~V}\right)$ et réfléchis par l'électrode $\mathrm{E}_{3}\left(V_{\mathrm{E}_{3}}=-10 \mathrm{~V}\right)$, le potentiel de $\mathrm{E}_{2}$ restant nul $\left(V_{\mathrm{E}_{2}}=0 \mathrm{~V}\right)$. Le remplissage terminé, le nuage d'électrons est alors " comprimé » en modifiant le potentiel de $E_{1}: V_{E_{1}}=-10 \mathrm{~V}$, afin d'empêcher les électrons d'atteindre à nouveau la pastille $P$. Les électrons piégés subissent alors des collisions avec échange de spin avec des atomes de potassium. Ils sont ensuite extraits en rendant $V_{\mathrm{E}_{3}}$ positif $\left(V_{\mathrm{E}_{3}}=+1500 \mathrm{~V}\right)$. La figure 9 donne l'évolution des

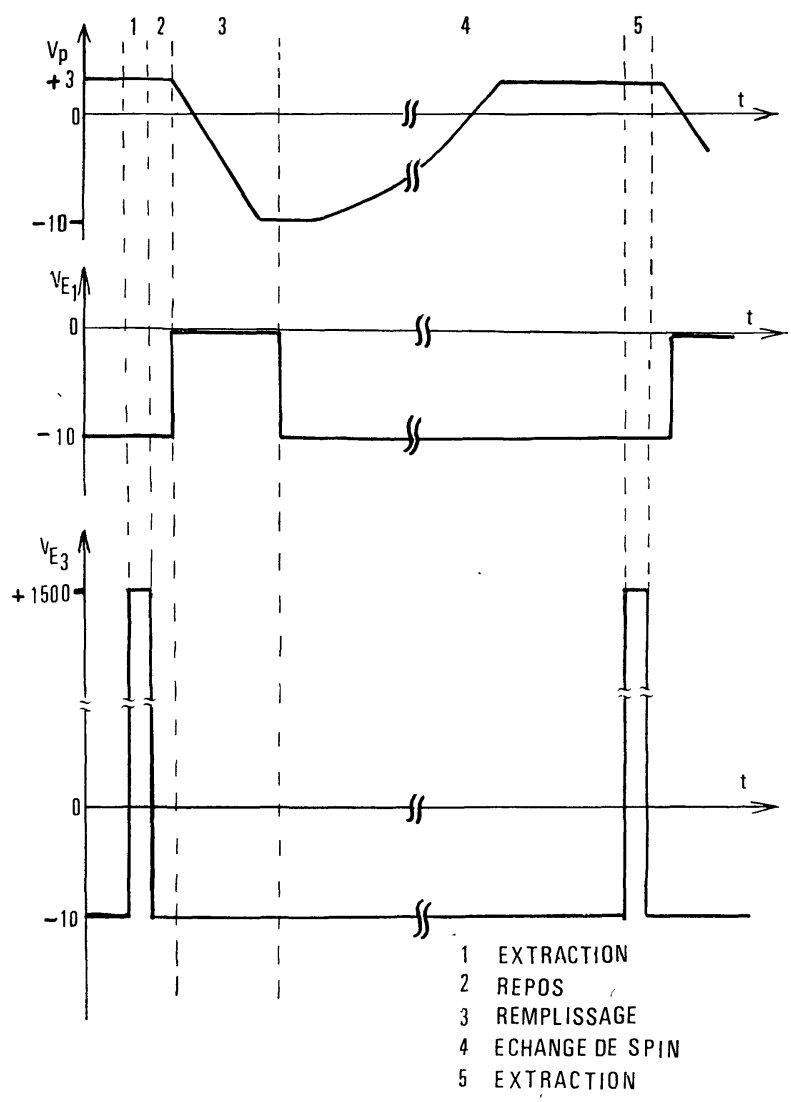

FIG. 9. - Schéma de variation des potentiels.

potentiels de $E_{1}, E_{2}, E_{3}$ et $P$ au cours du temps. Le problème est de savoir combien d'électrons peuvent être piégés dans un tel système.

5.1.2 Limitation due à la charge d'espace. - Calculons à partir de quelle densité d'électrons dans le piège, il ne sera plus possible d'injecter de nouveaux électrons secondaires.

Pour cela, supposons que le volume de rayon $a=0,5 \mathrm{~cm}$ (rayon de la pastille émissive) et de lon- gueur $L=20 \mathrm{~cm}$ (longueur du piège) contienne des électrons avec une densité $n$ constante. Calculons le potentiel $V_{\mathrm{c}}$ dû aux électrons au centre $C$ de ce nuage d'électrons. $V_{\mathrm{c}}$ est donné par la formule :

$$
\begin{aligned}
V_{\mathrm{c}}= & -\frac{n a^{2} e}{2}\left\{\frac{L}{2 a}\left[1+\left(\frac{L}{2 a}\right)^{2}\right]^{1 / 2}-\right. \\
& \left.-\left(\frac{L}{2 a}\right)^{2}-\log \left[\left(1+\left(\frac{L}{2 a}\right)^{2}\right)^{1 / 2}-\frac{L}{2 a}\right]\right\} .
\end{aligned}
$$

Supposant $a \ll L$, nous avons :

$$
V_{\mathrm{c}}=-9 \times 10^{-9} n a^{2}\left(0,5+\log \frac{L}{a}\right) .
$$

Considérant le cas précédent $(a=0,5 \mathrm{~cm}$; $L=20 \mathrm{~cm}$ ), nous obtenons :

$$
V_{\mathrm{c}}=9,5 \times 10^{-13} n:
$$

Supposons que les électrons secondaires ne quittent plus la pastille dès que $V_{\mathrm{c}}=-10 \mathrm{~V}$. (Le potentiel dû à la charge d'espace étant maximum en $C$, cette hypothèse nous donnera une limite inférieure du nombre d'électrons piégés.)

Cette hypothèse nous conduit à une population totale d'électrons de $1,65 \times 10^{8}$ et une densité $n=1,05 \times 10^{7}$ électrons $/ \mathrm{cm}^{3}$.

Supposons que les électrons aient une énergie moyenne de $5 \mathrm{eV}$, soit une vitesse $v=1,3 \times 10^{6} \mathrm{~m} / \mathrm{s}$. Nous pouvons espérer remplir ce piège en $1 \mu \mathrm{s}$ avec un courant de $170 \mu \mathrm{A}$. Ceci est facile à réaliser en utilisant le phénomène d'émission secondaire décrit plus haut. Cette limite ne pourrait être obtenue en utilisant une cathode chaude avec une tension accélératrice de $5 \mathrm{~V}$ [2], [16].

Comparons ce résultat avec la deuxième méthode décrite au paragraphe 4.1. Supposons $V_{R}=10 \mathrm{~V}$, soit donc $V_{0}=1,74 \mathrm{~V}$, et que le potentiel interne du faisceau varie de la façon suivante :

$$
V=\left(0,174+0,826\left(\frac{r}{a}\right)^{2}\right) V_{R}
$$

$a$ : rayon du faisceau.

(En réalité, il est difficile d'avoir une expression analytique de $V$, solution de l'équation différentielle :

$$
\frac{\mathrm{d}^{2} V}{\mathrm{~d} r^{2}}+\frac{1}{r} \frac{\mathrm{d} V}{\mathrm{~d} r}=\frac{A}{\sqrt{V}} \quad \text { (où } A \text { dépend de l'intensité) }
$$

mais l'expression ci-dessus nous suffit pour le calcul qui suit.)

Sachant que $L=0,2 \mathrm{~m}$, calculons le nombre d'électrons maximum que l'on peut piéger dans un volume de cette longueur :

$$
N=\frac{L}{e} \int_{0}^{a} \rho 2 \pi r \mathrm{~d} r
$$


avec

$$
\begin{aligned}
& \rho=\frac{I}{\pi a^{2} \sqrt{\frac{2 e}{m} \sqrt{V}}} \\
& V=\left(0,174+0,826\left(\frac{r}{a}\right)^{2}\right) V_{R} \\
& I=32,4 \times 10^{-6} V_{R}^{3 / 2}
\end{aligned}
$$

nous avons donc :

$$
N=4 \times 10^{8} L V_{R}
$$

ainsi, avec les valeurs prises ci-dessus, nous avons :

$$
N=8 \times 10^{8} \text { électrons . }
$$

Cette population serait obtenue théoriquement dans une induction magnétique infinie, tandis que la valeur précédemment trouvée en était une limite inférieure.

5.2 UNe AUTRE SOLUTION. - 5.2.1 L'injection. a) Description et fonctionnement du système d'injection. - Considérons un électron se déplaçant dans un champ électrique $\mathbf{E}$ et un champ magnétique $\mathbf{B}$ (E et $\mathbf{B}$ étant quelconques mais indépendants du temps: $\partial \mathbf{E} / \partial t=0$ et $\partial \mathbf{B} / \partial t=0)$. La force agissant sur cet électron est égale à :

$$
\mathbf{F}=-e(\mathbf{E}+\mathbf{V} \wedge \mathbf{B}) .
$$

Son mouvement peut se décomposer en écrivant que sa vitesse $\mathbf{v}$ est égale à :

$$
\mathbf{v}=\left(\mathbf{v}_{\|}+\mathbf{v}_{\perp}\right)+\mathbf{v}_{\mathrm{D}}
$$

les deux premiers termes représentent un mouvement quasi hélicoïdal autour des lignes de champ magnétique et $\mathbf{v}_{\mathbf{D}}$ représente la vitesse (appelée vitesse de dérive) du centre instantané de rotation de l'électron dans un plan perpendiculaire à $B$ en ce point.

Cette vitesse de dérive a pour expression :

$$
\mathbf{v}_{\mathrm{D}}=\frac{\mathbf{E} \wedge \mathbf{B}}{B^{2}}
$$

Considérons alors le condensateur schématisé sur la figure $10 a$ constitué de deux demi-cylindres d'axe $O z$ portés aux potentiels $+V_{0}$ et $-V_{0}$ dans un champ magnétique homogène parallèle à $O z$. La figure $10 b$ nous donne la trajectoire de différents électrons dans ce système supposé de longueur infinie en fonction du temps passé dans le condensateur. Remarquons que le rayon de giration des électrons étant inférieur à $1 \mathrm{~mm}$, la trajectoire moyenne des électrons s'appuie bien sur une surface équipotentielle.

Considérons alors le système à symétrie de révolution proposé sur la figure 11 . Il est constitué de deux miroirs $(\mathrm{A}, \mathrm{B}, \mathrm{C})$ et $(\mathrm{J}, \mathrm{K}, \mathrm{L}), V_{\mathrm{A}}=V_{\mathrm{L}}=-10 \mathrm{~V}$; $V_{\mathrm{B}}=V_{\mathrm{K}}=-5,5 \mathrm{~V}$ et $V_{\mathrm{C}}=V_{\mathrm{J}}=0 \mathrm{~V}$ destinés à retenir dans le piège des électrons d'énergie inférieure à $10 \mathrm{eV}$, et un système d'électrodes déflectrices

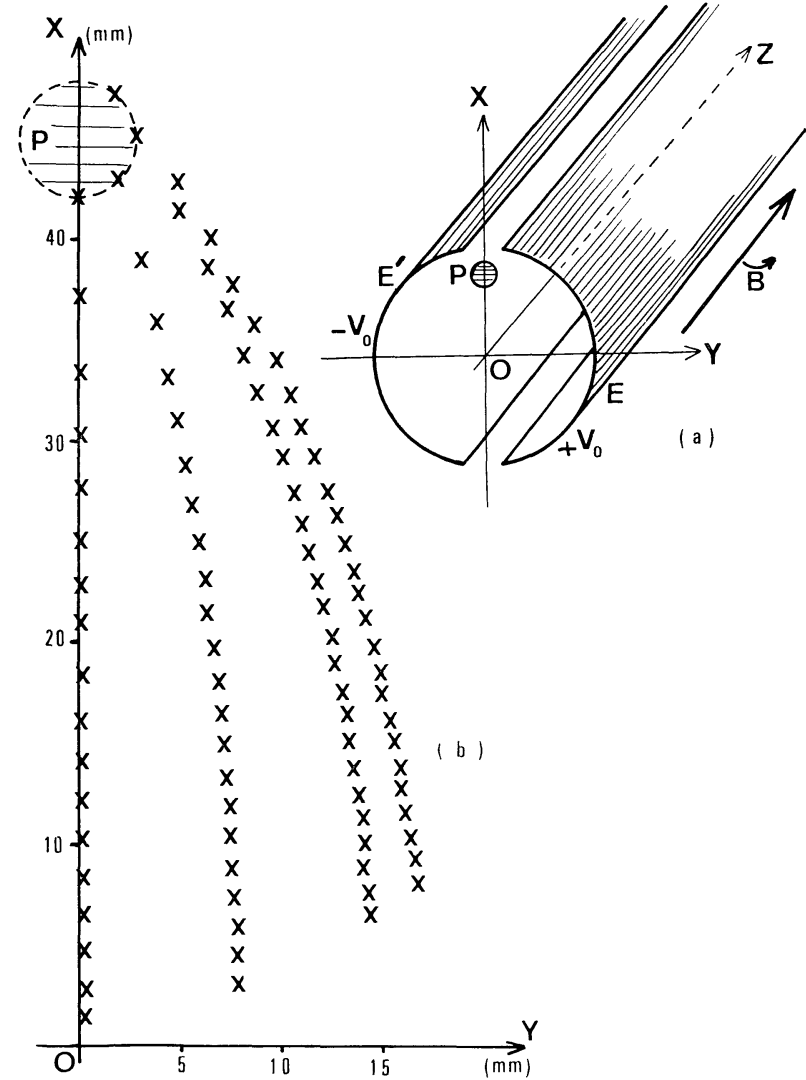

Fig. 10. - Trajectoires théoriques $(b)$ des électrons ayant $6 \mathrm{eV}$ d'énergie cinétique suivant $O z$ dans un condensateur $\left(\mathrm{E}, \mathrm{E}^{\prime}\right)$ (a) supposé infini et plongé dans une induction magnétique homogène $B=100 \mathrm{G}\left(V_{0}=15 \mathrm{~V}\right)$.

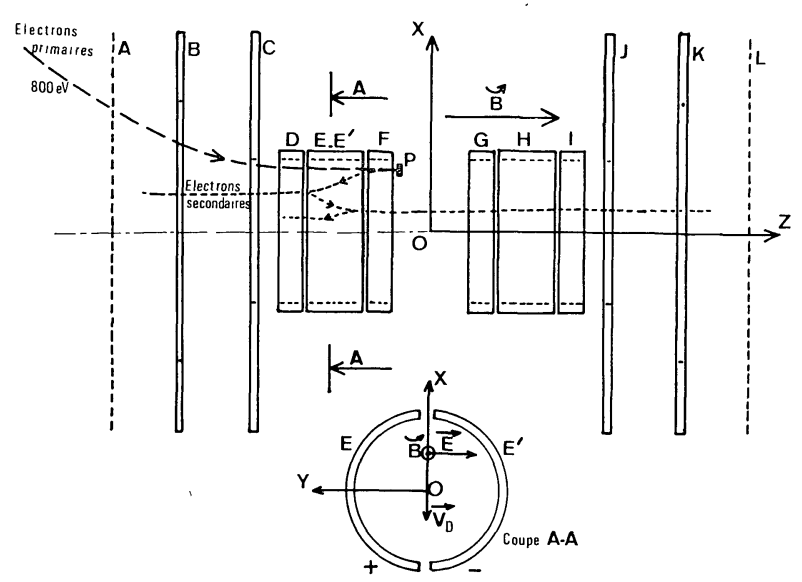

FIG. 11. - Schéma des électrodes.

$\left(\mathrm{D},\left(\mathrm{E}, \mathrm{E}^{\prime}\right), \mathrm{F}\right),\left(V_{\mathrm{D}}=V_{\mathrm{F}}=0 \mathrm{~V} ; V_{\mathrm{E}}=-30 \mathrm{~V}\right.$ et $\left.V_{\mathrm{E}^{\prime}}=+30 \mathrm{~V}\right)$ destiné à faire dévier les électrons secondaires vers l'axe du système. Le rôle des électrodes $(\mathrm{G}, \mathrm{H}, \mathrm{I}),\left(V_{\mathrm{G}}=V_{\mathrm{A}}=V_{\mathrm{I}}=0 \mathrm{~V}\right)$ identique aux électrodes $\left(\mathrm{D},\left(\mathrm{E}, \mathrm{E}^{\prime}\right), \mathrm{F}\right)$ est de respecter la symétrie des potentiels pendant la période de piégeage; elles n'interviennent pas lors de l'injection.

Le principe de l'injection est donc le suivant : un canon envoie des électrons de $800 \mathrm{eV}$ d'énergie sur une pastille $\mathrm{P}$ en platine au potentiel $V_{\mathrm{p}}=0 \mathrm{~V}$ les électrons 
secondaires rapides et les électrons primaires réfléchis vont frapper les parois ou reviennent vers le canon. Les électrons secondaires vrais, émis avec une énergie cinétique inférieure à $9 \mathrm{eV}$, sont déviés par les électrodes $\left(E, E^{\prime}\right)$, réfléchis par le miroir. $(A, B, C)$, déviés de nouveau par $\left(E, E^{\prime}\right)$ - ils évitent ainsi la pastille $P$, ce qui n'aurait pas été le cas si $V_{\mathrm{E}}=V_{\mathrm{E}^{\prime}}$ - réfléchis par $(J, K, L)$, déviés par $\left(E, E^{\prime}\right)$, et ainsi de suite. Les électrons sont donc peu à peu déviés vers l'axe du système.

b) Calcul des trajectoires d'injection sans charge d'espace. - La répartition de potentiel dans le piège, en l'absence de charge d'espace, a été calculée à l'aide d'une méthode de relaxation dérivée de l'équation de Laplace en coordonnées cartésiennes :

$$
\Delta V(x, y, z)=0 .
$$

On quadrille le volume à l'aide d'un réseau cubique dont les sommets sont repérés par $(I, J, K)$, le cube élémentaire ayant des dimensions aussi petites que l'on veut. On s'impose les potentiels sur les surfaces correspondant aux électrodes. Le potentiel en tout point (sauf sur les électrodes) est calculé par la formule suivante :

$$
V(I, J, K)=\frac{1}{6}\{V(I-1, J, K)+V(I+1, J, K)+\cdots\} .
$$

Ce calcul fait sur tout le volume étant répété autant de fois qu'on le désire pour obtenir la convergence voulue.

Les figures 12 et 13 montrent la forme de différentes équipotentielles pendant la période d'injection dans les plans $O x z$ et $O y z$.

Les trajectoires des électrons ont été intégrées à l'aide d'une méthode de Runge Kutta du 4e ordre [17] sur une machine IBM J 75. Les résultats sont en bon accord avec nos prévisions : les électrons se rapprochent bien de l'axe (Fig. 14), mais subissent une petite dérive due à la composante $E_{r}$ du champ électrique au moment de la réflexion (Fig. 15). Cette dérive peut être corrigée de trois façons différentes :

1) Modifier la répartition des potentiels des élec-

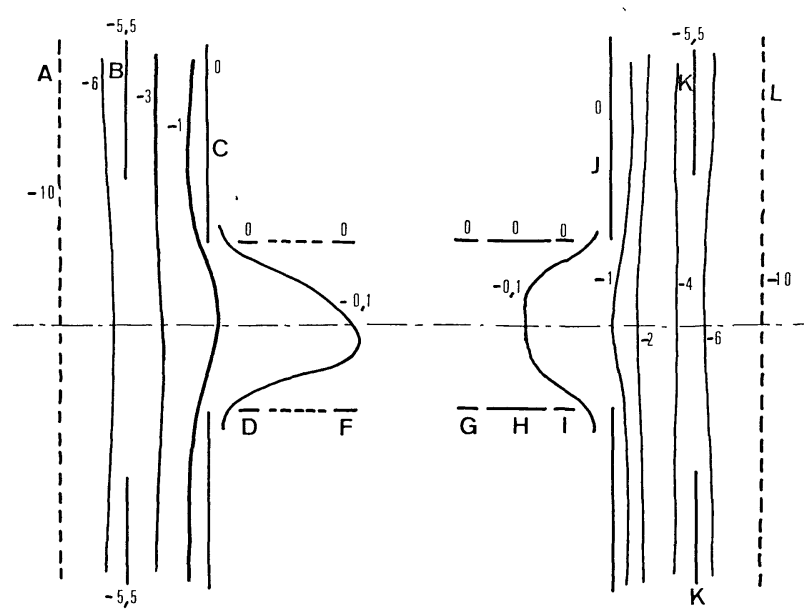

FIG. 12. - Tracé de quelques équipotentielles dans le plan $O x z$ pendant la période d'injection.

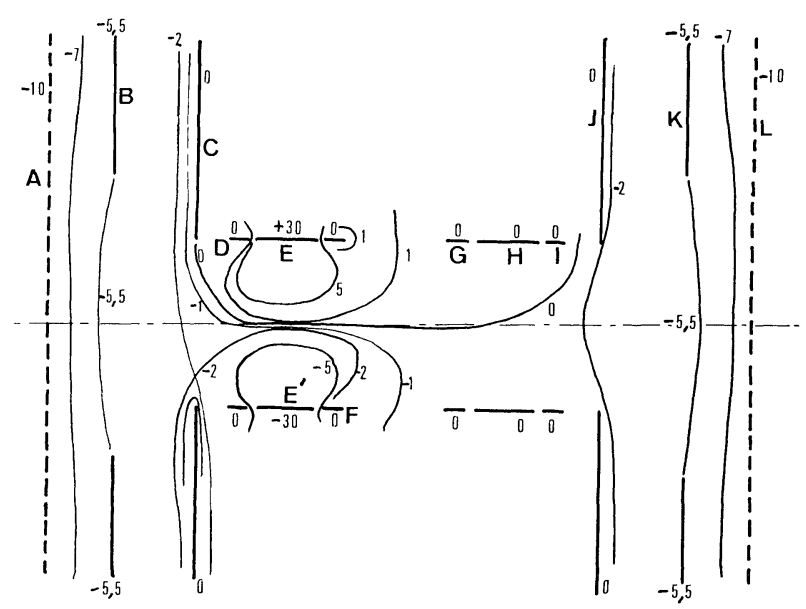

FIg. 13. - Tracé de quelques équipotentielles dans le plan $O y z$ pendant la période d'injection.

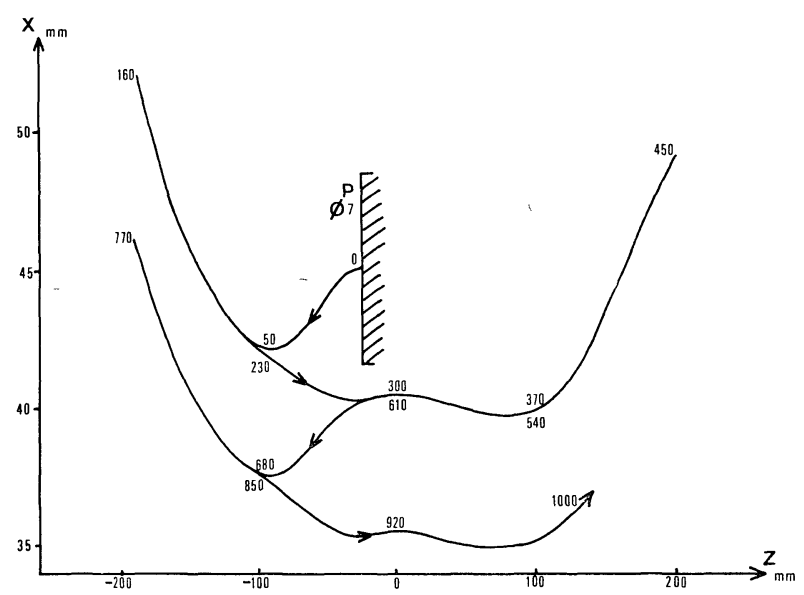

Fig. 14. - Projection de la trajectoire d'injection sur le plan $O x z$. Energie cinétique initiale $6 \mathrm{eV}$ suivant $O z$ et $V_{0}=20 \mathrm{~V}$.

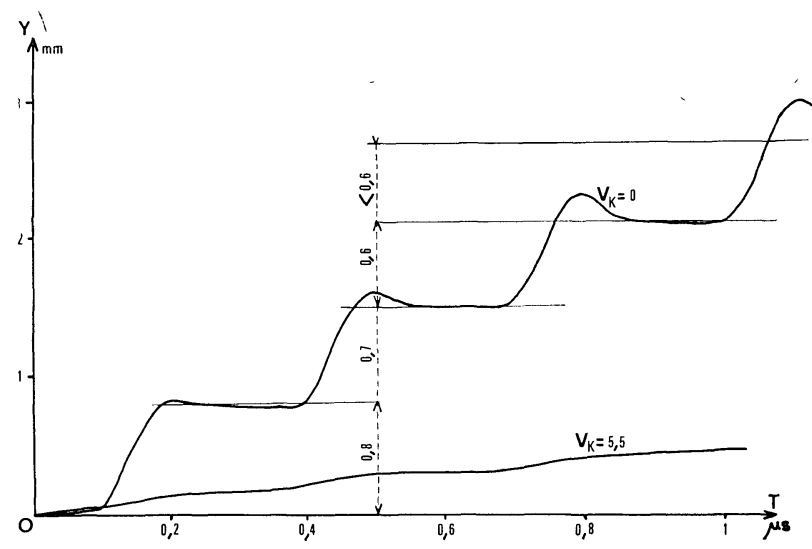

FIG. 15. - Projection de la trajectoire d'injection sur le plan $O y z$ (énergie cinétique initiale $6 \mathrm{eV}$ suivant $O z ; V_{0}=20 \mathrm{~V}$ ) dans deux cas :

1. $V_{\mathrm{K}}=V_{\mathrm{B}}=0 \mathrm{~V}$

2. $V_{\mathrm{K}}=V_{\mathrm{B}}=-5,5 \mathrm{~V}$.

trodes afin de minimiser $E_{r}$. (Remarquons que cette dérive diminue au fur et à mesure que l'électron se rapproche de l'axe.) 
2) Modifier le potentiel de la pastille, $V_{\mathrm{p}}$, afin que les électrons secondaires utiles $(E<10 \mathrm{eV})$ ne soient plus émis par une surface centrée sur l'axe $O x$, mais déplacé suivant $O y$ d'une grandeur convenable.

3) Remplacer l'électrode $\mathrm{H}$ par deux demi-cylindres $\mathrm{H}$ et $\mathrm{H}^{\prime}$ identiques aux électrodes $\mathrm{E}$ et $\mathrm{E}^{\prime}$, mais tournés de $90^{\circ}$ afin de donner aux électrons une dérive opposée à celle qu'ils ont lors des différentes réflexions.

Nous n'avons étudié que la première solution qui nous a donné des résultats convenables (Fig. 15). Nous voyons que la dérive des électrons est modifiée de façon très appréciable en ne changeant que le potentiel des électrodes B et $\mathbf{K}$.

Compte tenu de ces résultats, nous avons calculé le temps nécessaire d'injection (temps au bout duquel une quantité appréciable d'électrons atteignait l'axe du système) en comparant les résultats donnés sur la figure $10 b$ (donnant la déviation verticale en fonction du temps passé dans le condensateur supposé parfait) et la figure 14 (où nous avons noté le temps réel correspondant sur la trajectoire). Nous avons trouvé que les électrons atteignaient l'axe du système en un temps $t=14 \mu$ s. Nous pensons donc qu'au bout du temps $t_{i}=30 \mu \mathrm{s}$, le piège sera largement rempli.

5.2.2 Compression longitudinale; piégeage ; échange de spin. - Durant l'injection, les électrons oscillent sur une longueur de l'ordre de $40 \mathrm{~cm}$. Pour respecter les conditions énoncées au paragraphe 2.3, nous devons "comprimer » le nuage d'électrons afin d'empêcher les électrons d'atteindre des zones où la composante radiale du champ magnétique est trop importante.

Après l'injection, on comprime donc le faisceau en portant les électrodes A, B, C, J, K, L au potentiel $V=-20 \mathrm{~V}$ et les électrodes $\mathrm{D}, \mathrm{E}, \mathrm{E}^{\prime}, \mathrm{F}, \mathrm{G}, \mathrm{H}, \mathrm{I}$ au potentiel $V=0 \mathrm{~V}$. Nous obtenons alors une zone utile de piégeage de $20 \mathrm{~cm}$ de longueur pour des électrons de $6 \mathrm{eV}$ d'énergie (Fig. 16).

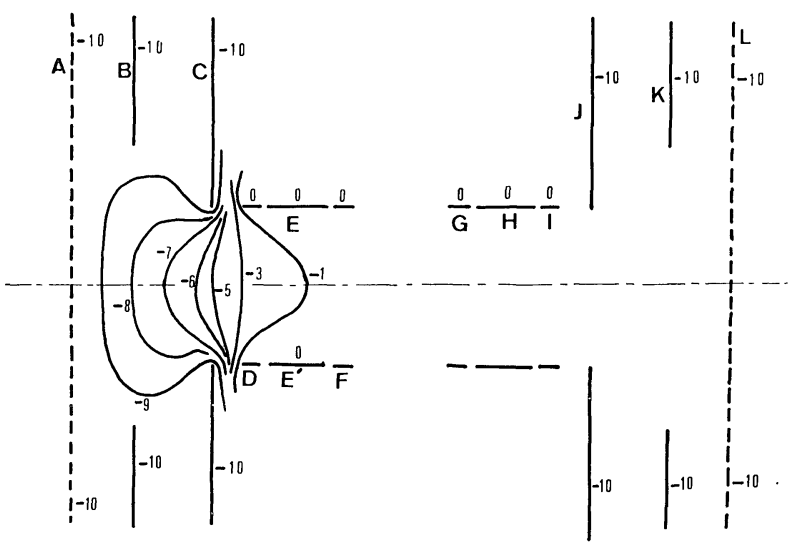

FIG. 16. - Tracé de quelques équipotentielles pendant la période de piégeage.

5.2.3 L'extraction. - On extrait les électrons en portant les électrodes $\mathrm{G}, \mathrm{H}, \mathrm{I}, \mathrm{J}, \mathrm{K}, \mathrm{L}$ au potentiel $V=+1500 \mathrm{~V}$. On mesure alors la polarisation du faisceau d'électrons extraits, d'énergie cinétique $1500 \mathrm{eV}$, par diffusion élastique sur des atomes de mercure [18] (Fig. 18).

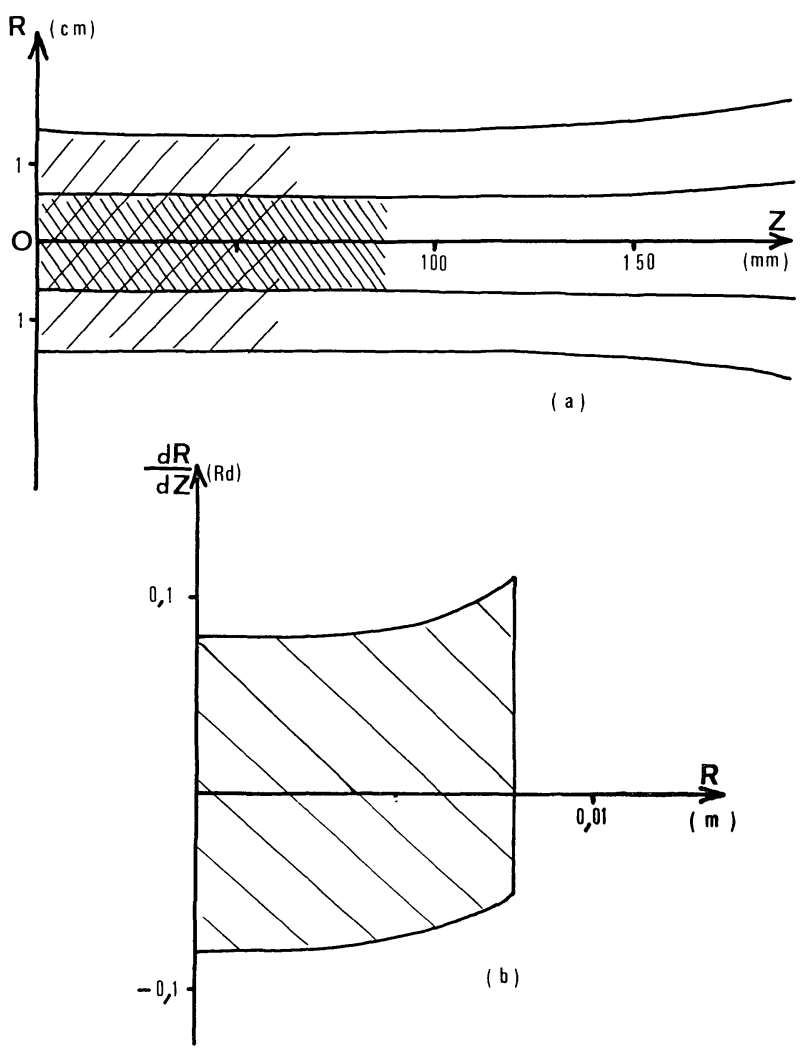

FIG. 17. - Trajectoires'à l'extraction (a) pour deux particules situées en $r=1,4 \mathrm{~mm}$ et $r=6 \mathrm{~mm}$. Emittance $(b)$ correspondante dans le cas où $r=6 \mathrm{~mm}$ en $z=200 \mathrm{~mm}=E=1,3 \mathrm{mRd}$.m.

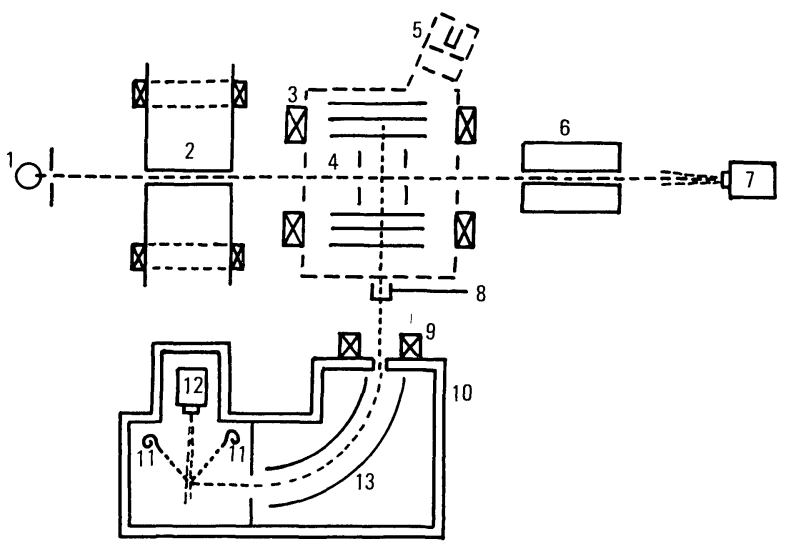

Fig. 18. - Vue d'ensemble de l'expérience :

1. Détecteur de Langmuir (mesure de la polarisation atomique).

2. Aimant dipolaire (analyseur).

3. Bobinages.

4. Piège contenant les électrodes de la figure 11 .

5. Canon à électrons $(800 \mathrm{~V})$.

6. Aimant hexapolaire (polariseur).

7. Four à potassium.

8. Cage de Faraday amovible.

9. Bobine de transfert.

10. Blindage.

11. Multiplicateurs tubulaires d'électrons pour la mesure de la polarisation des électrons. Ils sont en réalité dans un plan perpendiculaire à la figure.

12. Four à mercure.

13. Transformateur de polarisation. 
Durant son accélération, le faisceau voit un champ magnétique d'abord constant, puis décroissant. Ceci produit une légère divergence du faisceau qui s'accentue dans la région sans champ électrique (Fig. 17). Pour le faire reconverger, on place une bobine centrée sur l'axe, dont les dimensions sont petites afin de ne pas modifier le champ magnétique dans la zone de piégeage proprement dite. Au niveau de cette bobine, les lignes de champ se rapprochent de l'axe, après la bobine, elles s'épanouissent dans un blindage situé à l'entrée du système de détection.

La bobine de focalisation commande l'ouverture angulaire du faisceau à l'entrée du système de détection. On ajustera expérimentalement le courant excitateur pour que celle-ci ait la valeur choisie.

Nous avons résumé dans le tableau ci-dessous le fonctionnement de l'ensemble en donnant l'évolution des tensions appliquées aux diverses électrodes au cours d'un cycle.

\begin{tabular}{|c|c|c|c|}
\hline Electrode & $\begin{array}{l}\text { Injection } \\
(30 \mathrm{~s})\end{array}$ & $\begin{array}{l}\text { Piégeage } \\
(1000 \mathrm{~s})\end{array}$ & $\begin{array}{c}\text { Extraction } \\
(0,4 \text { s })\end{array}$ \\
\hline - & - & - & - \\
\hline A & -10 & -20 & $-\quad 20$ \\
\hline B & $-\quad 5,5$ & -20 & 20 \\
\hline $\mathrm{C}$ & 0 & -20 & 20 \\
\hline $\mathrm{D}$ & 0 & 0 & 0 \\
\hline $\mathrm{E}$ & -30 & 0 & 0 \\
\hline $\mathrm{E}^{\prime}$ & +30 & 0 & 0 \\
\hline $\mathrm{F}$ & 0 & 0 & 0 \\
\hline G & 0 & 0 & +1500 \\
\hline $\mathrm{H}$ & 0 & 0 & +1500 \\
\hline I & 0 & 0 & +1500 \\
\hline J & 0 & -20 & +1500 \\
\hline $\mathrm{K}$ & $-\quad 5,5$ & -20 & +1500 \\
\hline $\mathrm{L}$ & -10 & -20 & +1500 \\
\hline
\end{tabular}

En pratique, il est commode d'avoir le système de détection de polarisation au potentiel de la masse, les potentiels réels seront donc inférieurs de $1500 \mathrm{~V}$ à ceux indiqués.

5.2.4 Limitation due à la charge d'espace. Performances. - Considérons que la surface utile de la pastille émissive ait $2 \mathrm{~mm}$ de largeur (ceci afin d'obtenir des électrons d'énergie cinétique inférieure à à $9 \mathrm{eV}$ ), et qu'elle soit circulaire. Calculons alors l'intensité des électrons secondaires émis. Pour cela faisons un calcul analogue à celui du paragraphe 5.1.2.

$$
V_{\mathrm{c}}=-9 \times 10^{-9} n a^{2}\left(0,5+\log \frac{L}{a}\right)
$$

prenant $a=1 \mathrm{~mm}$ et $L=20 \mathrm{~cm}$, nous obtenons :

$$
V_{\mathrm{c}}=-5,2 \times 10^{-14} n
$$

ce-qui correspond lorsque $V_{\mathrm{c}}=-10 \mathrm{~V}$ à une densité d'électrons

$$
n=1,9 \times 10^{8} \text { électrons } / \mathrm{cm}^{3} .
$$

Considérant des électrons d'énergie moyenne $5 \mathrm{eV}$, donc de vitesse $v=1,35 \times 10^{6} \mathrm{~m} / \mathrm{s}$, ceci correspond à un courant d'électrons secondaires d'intensité

$$
I_{\mathrm{s}}=125 \mu \mathrm{A}
$$

le temps d'injection est $t_{\mathrm{i}}=30 \mu \mathrm{s}$, mais le temps pour que les électrons atteignent l'axe du système est de $14 \mu \mathrm{s}$. Le nombre correspondant d'électrons injectés dans un volume de longueur $L=20 \mathrm{~cm}$ et de section triangulaire de $5 \mathrm{~cm}^{2}$ (hauteur $5 \mathrm{~cm}$, base $2 \mathrm{~cm}$ ), est donc :

$$
N_{t}=11 \times 10^{9} \text { électrons . }
$$

Supposons que ces électrons soient répartis uniformément, si nous extrayons les électrons compris dans un volume de longueur $L=20 \mathrm{~cm}$ et de $1 \mathrm{~cm}^{2}$ de section, en 0,4 , s nous aurons une intensité d'électrons polarisés de :

$$
I_{\text {extrait }}>1 \mathrm{~mA}
$$

nombre d'électrons extraits par pulse $>2,5 \times 10^{9}$.

Nous gagnons donc un facteur 15 par rapport à la solution précédente.

Nous aurons un courant moyen (le système fonctionnant à une fréquence voisine de $1000 \mathrm{~Hz}$ ) :

$$
I_{\text {moyen }}=0,4 \mu \mathrm{A} \text {. }
$$

$\mathrm{Ce}$ calcul sous-estime plusieurs paramètres : $I_{\mathrm{s}}=125 \mu \mathrm{A}$ est une limite inférieure du courant d'électrons secondaires; la densité des électrons est plus forte au voisinage de l'axe ; le faisceau au niveau de l'axe aura une largeur inférieure à $2 \mathrm{~cm}$. On peut donc penser obtenir un courant plus important. Des mesures seront effectuées en faisant varier l'énergie moyenne des électrons, ceci en sélectionnant les électrons au moment de l'injection.

5.2.5 Vue d'ensemble de l'expérience. - Ce système peut donc fournir une quantité importante d'électrons de très basse énergie qui remplissent toutes les conditions d'échange de spin avec des atomes de potassium polarisés. La figure 18 donne un schéma de l'expérience.

Le jet atomique, de polarisation connue, est produit par passage d'un faisceau d'atomes de potassium dans un hexapôle [7]. Après traversée du faisceau d'électrons, on peut mesurer la perte de polarisation du jet atomique à l'aide d'un analyseur dipolaire associé à un détecteur de Langmuir [19].

Les électrons piégés passent après excitation dans un déflecteur électrostatique qui rend leur polarisation transversale, ce qui permet d'en effectuer la mesure par détection de l'asymétrie dans la diffusion élastique des électrons par des atomes de mercure [18]. Contrairement à la diffusion de Mott sur cible d'or, qui exige des énergies d'électrons de 80 à $120 \mathrm{keV}$, ce procédé permet de connaître la polarisation avec des énergies de l'ordre de $\mathrm{keV}$, ce qui est extrêmement intéressant du point de vue expérimental. 
La comparaison entre la perte de polarisation du jet d'électrons en fonction de leur énergie cinétique fournira d'utiles renseignements sur les conditions propres à assurer un bon transfert de polarisation et sur les causes éventuelles de dépolarisation.

6. Conclusion. - Le courant crête obtenu, supérieur à $1 \mathrm{~mA}$, doit permettre d'utiliser un tel faisceau dans un accélérateur linéaire pour réaliser diverses expériences de physique nucléaire ou atomique.

En conclusion, citons-en quelques-unes proposées par divers auteurs :

a) La diffusion élastique d'électrons non polarisés de grande énergie par des protons a permis de découvrir les propriétés de structure des protons en admettant la validité de la théorie de la diffusion et les approximations dans l'expression de la section efficace de diffusion.

$\mathrm{Si}$ on observe la polarisation de protons diffusés élastiquement par des électrons polarisés, trois nouvelles mesures indépendantes sont possibles : $P_{x}, P_{y}$, $P_{z}$. La connaissance de $P_{z}$ (polarisation longitudinale) donne un nouveau moyen d'examiner la validité de la conservation de la parité et de connaître l'influence des approximations faites en utilisant des électrons non polarisés. La mesure de $P_{x}$ et $P_{y}$ donnerait le rapport des facteurs de forme électrique et magnétique. Elle permettrait une vérification supplémentaire de la validité de la formule de Rosenbluth [20], [21].

b) La diffusion élastique d'électrons polarisés par une cible de deutérons ou de noyaux polarisés permettrait la séparation des facteurs de forme électrique de charge, électrique quadrupolaire et magnétique dipolaire [22].

c) L'étude de la diffusion inélastique d'électrons polarisés sur une cible de protons polarisés permettrait de déduire la partie imaginaire de l'amplitude "spinflip » dans la diffusion photon virtuel-proton s'il y a électro-production d'un méson [23].

d) On peut utiliser les électrons polarisés pour exciter des noyaux polarisés et déterminer ainsi les éléments de la matrice nucléaire réduite [20].

e) Une autre utilisation serait la production par Bremstrahlung de photons polarisés linéairement ou circulairement.

\section{ANNEXE}

\section{CALCUL DE LA DÉPOLARISATION DUE A LA COMPOSANTE RADIALE DU CHAMP MAGNÉTIQUE}

On considère des électrons dans un piège magnétique défini par les paramètres suivants :

- champ magnétique $H_{z}$ au centre : $125 \mathrm{Oe}$,

- longueur du piège : $20 \mathrm{~cm}$,

$5 \mathrm{eV}$,

- énergie cinétique de translation des électrons:

- temps mis par un électron pour faire un allerretour : $\tau=3 \times 10^{-7} \mathrm{~s}$.

L'électron voit un champ $H_{z}$ pratiquement constant auquel est superposé un champ radial oscillant $H_{r}(t)$ à la fréquence $w=2 \pi / \tau$.

L'évolution de la polarisation est déterminée par un calcul de perturbation. L'équation d'évolution s'écrit :

$$
\frac{i h}{2 \pi} \frac{\mathrm{d}}{\mathrm{d} t} \rho=[\mathrm{He}, \rho]
$$

$h$ est la constante de Planck,

$\rho$ est la matrice densité des électrons,

He est l'hamiltonien de perturbation dû au champ radial ; il s'écrit :

$$
\mathcal{H}=\left(\frac{h}{4 \pi}\right) \cdot\left(w_{z} \cdot \sigma_{z}+w_{x} \cdot \sigma_{x}+w_{y} \cdot \sigma_{y}\right)
$$

avec

$$
w_{i}=-\frac{4 \pi \mu H_{i}}{h} \quad(i=x, y, z)
$$

$\mu$ est le magnéton de Bohr.

$\sigma_{x}, \sigma_{y}, \sigma_{z}$ sont les matrices de Pauli.
On a également :

$$
\rho=\left[\begin{array}{ll}
1+P_{z} & P_{x}-i P_{y} \\
P_{x}+i P_{y} & 1-P_{z}
\end{array}\right] .
$$

En reportant dans l'éq. (1), on arrive au système d'équations :

$$
\begin{aligned}
& \frac{\mathrm{d} P_{x}}{\mathrm{~d} t}=-w_{z} P_{y}+w_{y} P_{z} \\
& \frac{\mathrm{d} P_{y}}{\mathrm{~d} t}=w_{z} P_{x}-w_{x} P_{z} \\
& \frac{\mathrm{d} P_{z}}{\mathrm{~d} t}=w_{x} P_{y}-w_{y} P_{x} .
\end{aligned}
$$

Posant : $P_{r}=P_{x}+i P_{y}$ et $w_{r}=w_{x}+i w_{y}$, le système d'équations se ramène à deux équations :

$$
\begin{aligned}
& \frac{\mathrm{d} P_{z}}{\mathrm{~d} t}=\left(\frac{i}{2}\right)\left(w_{r} \cdot P_{r}^{*}-w_{r}^{*} \cdot P_{r}\right) \\
& \frac{\mathrm{d} P_{r}}{\mathrm{~d} t}=i\left(w_{z} \cdot P_{r}-w_{r} \cdot P_{z}\right)
\end{aligned}
$$

(3) s'intègre sous la forme :

$$
P_{r}=-i \cdot \exp \left(i w_{z} t\right) \cdot \int_{0}^{t} w_{r} \cdot P_{z} \cdot \exp \left(-\mathrm{i} w_{z} t\right) \cdot \mathrm{d} t
$$


$w_{r}$ peut s'écrire :

$$
w_{r}=W \cdot \exp (-i w t) .
$$

Reportant dans (4) et intégrant par parties, on a :

$$
\begin{aligned}
P_{r}=\frac{w}{w+w_{z}} & \cdot\left\{\sum_{n=0}^{\infty} \frac{(i)^{n}(-1)^{n}}{\left(w+w_{z}\right)^{n}} \times\right. \\
& \left.\times\left(\exp (-i w t) \cdot P_{z}^{(n)}-\exp \left(i w_{z} t\right) \cdot P_{z_{0}}^{(n)}\right)\right\}
\end{aligned}
$$

$P_{z}^{(n)}$ est la dérivée d'ordre $n$ de $P_{z}$ par rapport au temps, $P_{z_{0}}^{(n)}$ est la dérivée d'ordre $n$ de $P_{z}$ au temps $t=0$.

Connaissant $P_{r},(2)$ peut s'écrire :

$$
\begin{aligned}
& \frac{\mathrm{d} P_{z}}{\mathrm{~d} t}= \frac{W^{2} i}{2\left(w+w_{z}\right)} \cdot \sum_{n=0}\left\{(-1)^{n} P_{z}^{(n)} \frac{(-i)^{n}-(i)^{n}}{\left(w+w_{z}\right)^{n}}+\right. \\
&+\frac{(-1)^{n} \cdot P_{z_{0}}^{(n)}}{\left(w+w_{z}\right)^{n}} \cdot\left[(i)^{n} \cdot \exp \left(i w+i w_{z}\right) t-\right. \\
&\left.\left.-(-i)^{n} \cdot \exp \left(-i w t-i w_{z} t\right)\right]\right\}
\end{aligned}
$$

soit :

$$
\begin{aligned}
\frac{\mathrm{d} P_{z}}{\mathrm{~d} t}=W^{2} \sum_{n=0} \frac{(-1)^{n}}{\left(w+w_{z}\right)^{n+1}} & \cdot\left[P_{z}^{(n)} \cdot \sin \left(\frac{n \pi}{2}\right)-P_{z_{0}}^{(n)} \times\right. \\
& \left.\times \sin \left(w t+w_{z} t+\frac{n \pi}{2}\right)\right] .
\end{aligned}
$$

Supposons nulles les dérivées successives de $P_{z}$ à $t=0$ et intégrons une fois :

$$
\begin{aligned}
P_{z}\left[1+\frac{\left(w+w_{z}\right)^{2}}{W^{2}}\right]+\sum_{n=1}^{\infty} \frac{(-1)^{n} P_{z}^{(2 n)}}{\left(w+w_{z}\right)^{2 n}}= \\
=P_{z_{0}}\left[\frac{\left(w+w_{z}\right)^{2}}{W^{2}}+\cos \left(w t+w_{z} t\right)\right] .
\end{aligned}
$$

Une solution particulière est :

$$
\begin{aligned}
P_{z}=\frac{P_{z_{0}} \cdot W^{2}}{W^{2}+\left(w+w_{z}\right)^{2}} \cdot \cos (w & \left.+w_{z}\right) t+ \\
& +P_{z_{0}} \frac{\left(w+w_{z}\right)^{2}}{W^{2}+\left(w+w_{z}\right)^{2}} .
\end{aligned}
$$

Cette solution devient la solution générale si on néglige les dérivées du $3^{\mathrm{e}}$ ordre. On a alors :

$$
\frac{\Delta P_{z}}{P_{z_{0}}}=-\frac{2 W^{2}}{W^{2}+\left(w+w_{z}\right)^{2}} \cdot \sin ^{2}\left(w+w_{z}\right) \frac{t}{2}
$$

$W$ et $w, w_{z}$ si bien qu'on peut écrire :

$$
\frac{\Delta P_{z}}{P_{z_{0}}}<\frac{2 W^{2}}{w_{z}^{2}}=\frac{2\left|H_{r_{\max }}\right|^{2}}{\left|H_{z}\right|^{2}} .
$$

Si on veut $\Delta P_{z} / P_{z_{0}}<10^{-3}$, on doit avoir

$$
H_{r}<2,2 \times 10^{-2} H_{z}
$$

soit pour $H_{z}=125$ Oe, $H_{r}<2,8 \mathrm{Oe}$.

\section{Bibliographie}

[1] Capdequi-Peyranere (P.), Rap. CEA R 3197, EUR 3437 f, 1967.

[2] Byrne (J.), Farago (P. S.), Proc. Phys. Soc., 1965, 86, 801.

[3] Barbier (M.), Rap. CERN 61-23, 1961.

[4] Wilmers (M.) et al., Z. Phys., 1969, 27, 3, 5204.

[5] Krisciokaitis (R, J.), Wu YaNG Tsai, Nucl. Instr. Meth., 1970, 83, 45.

[6] Coiffet (Ph.), Gilles (J. P.), Checkrahamatoula, C. R. Acad. Sci. Paris, 1969, 269, 1177.

[7] Colffet (Ph.), J. Physique, 1971, 32, 113.

[8] Dehmelt (H. G.), J. Physique et le Radium, 1958, 19, 866.

[9] Rubin (K.) et al., Phys. Rev., 1960, 117, 1, 151.

[10] Balling (L. C.), Phys. Rev., 1966, 151, 1, 1.

[11] Mott (N. F.), Massey (H.), "The atomic collisions ", Clarendon Press, Oxford, 1965.

[12] Montgomery (D. B.), Rap. AFOSR, 1525-MIT, Cambridge, 1962.

[13] GiraRd (B.), Nucl. Instr. Meth., 1964, 25, 269.
[14] Pierce (J. R.), Theory and Design of Electron beams D. Van Nostrand Company, 1954.

[15] Bruining (H.), «Physics and Application of secondary electron emission », Pergamon Press, London, 1954

[16] Farago (P. S.) Siegmann (H. C.), Phys. Lett., 1966, 20, 3, 279.

[17] Ralston (A.), Mathematical methods of digital computers, J. Wiley and Sons Publishers, 1960, 95-109.

[18] Kessier (J.), Rev. of Mod. Phys., 1969, 41, 1, 3-25.

[19] Gilles (J.-P.), Coiffet (Ph.), Brebec (J.-M.), Etude et mesure des caractéristiques et de la polarisation d'un jet atomique orienté. A paraître dans Nucl. Instr. Meth.

[20] Hughes (V. W.), Phys. Rev. Lett., 1965, 15, $1,1$.

[21] Bounin (P.), Rap. LNF 65/22 CNEM Frascati, 1965.

[22] Schildnecht (D.), Proc. of the Int. Symp. on the electrons and photons on high energy, Hambourg, juin 1965,81 .

[23] Iddings (C. K.), Phys. Rev., 1965, 138B, 446. 